\title{
An Improved Image Segmentation Algorithm CT Superpixel Grid Using Active Contour
}

\author{
Yuntao Wei and Xiaojuan Wang $(\mathbb{D}$ \\ College of Electronic Information Technology, Jiamusi University, Jiamusi 154007, China \\ Correspondence should be addressed to Xiaojuan Wang; wangxiaojuan@jmsu.edu.cn
}

Received 9 April 2021; Revised 12 May 2021; Accepted 21 May 2021; Published 4 June 2021

Academic Editor: Wenqing Wu

Copyright (c) 2021 Yuntao Wei and Xiaojuan Wang. This is an open access article distributed under the Creative Commons Attribution License, which permits unrestricted use, distribution, and reproduction in any medium, provided the original work is properly cited.

\begin{abstract}
The traditional CT image segmentation algorithm is easy to ignore image contour initialization, which leads to the problem of long time consuming and low accuracy. A superpixel mesh CT image improved segmentation algorithm using active contour was proposed. CT image superpixel gridding was carried out first; secondly, on the basis of gridding, the region growth criterion was improved by superpixel processing, the region growth graph was established, the image edge salient graph was calculated based on the growth graph, and the target edge was obtained as the initial contour; finally, the Mumford-Shah model in the active contour model was improved; the energy functional was constructed based on the improved model and transformed into the symbol distance function. The results show that the proposed algorithm takes less time to mesh superpixels, the accuracy of image edge calculation is high, the correct classification coefficient is as high as 0.9 , and the accuracy of CT image segmentation is always higher than $90 \%$, which has superiority.
\end{abstract}

\section{Introduction}

Computed tomography (CT) has the advantages of fast imaging and high image resolution. It is an important electronic imaging technology $[1,2]$. As an important detection method in routine clinical examinations, it has become a computer-assisted human organ examination, and followup an important basis for medical treatment is currently widely used in clinical medicine $[3,4]$. In the computeraided diagnosis medical system, segmentation of the region of interest in the CT image is an important step in diagnosis and the key technical support for subsequent 3D image reconstruction. It plays an important role in the precise diagnosis and treatment of diseases and can also reduce calculations to a certain extent [5]. Human CT scan images include multiple organs such as the chest, abdomen, and blood vessels. In view of the complexity, irregularity, and uncertainty of human organs [6], it brings certain difficulties to image segmentation, and ordinary image segmentation techniques are difficult to obtain more accurate. As a result, the limitations are strong, so there is an urgent need to find an effective CT image segmentation algorithm, which is of great significance for clinical auxiliary diagnosis and quantitative analysis with the help of CT images [7].

Traditional image segmentation algorithms mostly use pixels as the basic unit and represent the pixels by forming a matrix, but it is often easy to ignore the internal connection between pixels and cannot comprehensively consider pixel characteristics. Segmentation will occur when there is occlusion or low texture [8]. The basic principle of the concept of superpixel is to judge the similarity according to the texture features and brightness of the pixels. Therefore, the pixels can be divided into different subregions, which is convenient for subsequent calculation, improves the efficiency of image segmentation, and makes up for the shortcomings of traditional image segmentation algorithms [9]. Taha and Hanbury propose an efficient evaluation tool implementing the 20 selected metrics. The tool is optimized to perform efficiently in terms of speed and required memory, also if the image size is extremely large as in the case of whole body MRI or CT volume segmentation [10]. Chengcheng et al. [11] used convolutional neural network to extract image features, retrieved the image set, and used Gaussian kernel density estimation to weight the superpixels of the image, 
thereby improving the pixel matching accuracy and completing high-precision image segmentation; Jiarui et al. [12] perform clustering analysis based on the results of superpixel segmentation and perform superpixel segmentation on the image area to obtain matching areas containing feature points. Based on this, effective feature points can be obtained to help subsequent analysis. Superpixel segmentation is used in this algorithm. Miao et al. [13] improved and analyzed the current superpixel method. First, iteratively clustered images based on local information performed the first superpixel segmentation on the clustered images and then performed iterative segmentation again based on the color standard deviation and merge to obtain an effective superpixel segmentation algorithm.

With the help of the concept of superpixel segmentation, this paper proposes an improved segmentation algorithm for superpixel grid CT images using active contours. The main contributions are as follows: (1) Using superpixels to improve the region growth criterion, the determination of the initial contour is more accurate. (2) The paper improves the Mumford-Shah model, fully considers the pixel texture characteristics, and increases the accuracy of CT image segmentation. (3)Various types of CT image data are used for experimental analysis, and the selection of experimental indicators is abundant, which greatly increases the validity of the experimental results.

\section{Related Work}

For CT image segmentation, many studies have been carried out at home and abroad. Literature [14] combines convolutional neural network and superpixel analysis for CT image segmentation, uses the superpixel method to grid the CT image while labeling the label, and uses the convolutional neural network to train the superpixel edge to complete the rough image segmentation. The segmented edge is used as the initial contour to be segmented again to obtain accurate segmentation results, but the algorithm has certain shortcomings in terms of running time; literature [15] also uses the superpixel method for image gridding and convolutional neural network for image edge extraction. The extracted edge pixels are formed as the initial contour and then combined with the integrated energy function to complete the image segmentation. The accuracy of the algorithm needs to be improved; the literature [16] combines superpixel segmentation and fuzzy C-means clustering to perform image segmentation. Use spatial neighborhood information to enhance image grayscale, use morphological knowledge to remove image peripheral influencing factors, and then perform superpixel classification on the grayscale and texture features of the image to obtain a more accurate image segmentation result, but for image pixel blocks underutilized.

In foreign studies, the literature [17] proposed a new three-dimensional adaptive active contour method for CT lung image segmentation. The method starts from a sphere in the lung. The force acting on the sphere is divided into the lung. This process is performed iteratively in order to minimize the energy function related to the $3 \mathrm{D}$ deformation model. The experimental results show the superiority of this algorithm in CT image segmentation, but the use of active contours needs to be improved; the literature [18] that proposed an image segmentation algorithm based on superpixel clustering is proposed. First, the algorithm uses superpixel preprocessing technology to quickly divide the image into a certain number of superpixel regions with specific information and then uses the similarity matrix to provide input to the spectral clustering algorithm information. The superpixel area is clustered, and the final image segmentation result is obtained, but the segmentation accuracy is not high. Literature [19] focuses on simple linear iterative clustering superpixels and fast and automatically adjustable Gaussian path The image segmentation technology combined with the basis function kernel fuzzy C-means combines superpixels and image segmentation as a preprocessing step for image classification and then provides better results for image classification, but the accuracy of the algorithm is not high [20].

In order to obtain more accurate CT image segmentation results, this paper proposes an improved segmentation algorithm for CT images based on active contour superpixel grid. First, the CT image is processed with superpixel grids, and then based on the concept of superpixels, the region growth criterion is improved, and the concept of center-surround contrast is introduced to calculate the prominent area of the image edge, and the initial contour is obtained. The contour initialization problem of image segmentation is well completed. The active contour model is used to complete the segmentation of superpixel grid CT images. The experiment selects two data sets, TCIA and DeepLesion, as data sources and uses lung CT images, brain CT images, and vertebra CT images for analysis. The results show that the proposed algorithm is excellent in time-consuming, correct classification, and segmentation accuracy. Compared with other algorithms, it provides data support for further research on CT images.

\section{CT Image Superpixel Gridding}

In order to effectively complete the CT image segmentation, it is first necessary to perform the CT image superpixel gridding process, so that the pixels of the same pixel block in the image have a high degree of similarity in terms of color, texture, and intensity [21].

First, initialize the CT image pixels. The random collection of pixel seed points is represented as $D=\left\{d_{1 n}, d_{2 n}, \cdots, d_{k n}\right\}$ a grid with $2 \times 2$ units, the seed points are evenly distributed in the grid, and the pixels in the neighborhood of the seed points are marked. Expression:

$$
\left\{\begin{array}{l}
d(q)=\left(x_{q}, y_{q}\right)=1, \\
d_{k}(q)=\left(x_{k q}, y_{k q}\right)=\infty,
\end{array}\right.
$$

where $\left(x_{q}, y_{q}\right)$ represents a certain gray pixel in the neighborhood.

The original grayscale $D$ pixel of the seed point is expressed as $\left(x_{n}, y_{n}\right)$; in the grid area, all the pixels $D$ in the neighborhood of the seed point are counted, and the distance 
between the seed point $D$ and the pixel point is calculated separately, and the calculation is based on the grayscale feature. The formula is as follows:

$$
s(p, q)=\sqrt{\left(x_{p}-x_{q}\right)^{2}+\left(y_{p}-y_{q}\right)^{2}}
$$

where $s(p, q)$ represents the spatial distance of grayscale features between two pixels.

Calculated the straight $D$ line distance between the seed point and the pixel point, expressed as:

$$
s^{\prime}(p, q)=\sqrt{\left(x_{p}-x_{q}\right)^{2}}
$$

The linear distance and the spatial distance are combined to synthesize a single unit of measure $G$. The formula is as

$$
G=\sqrt{\left(\frac{s^{\prime}}{\partial}\right)^{2}+\left(\frac{s}{B}\right)^{2}}
$$

where $\partial$ represents the balance coefficient of the pixel gray feature in the straight line and $B$ represents the step length between different seed points, which can be calculated by the following formula:

$$
B=\sqrt{\frac{N}{L}}
$$

where $N$ means that the CT image contains the number of $L$ pixels and the number of pixel blocks to be divided.

\section{Target Edge Calculation in CT Image Using Superpixel Region Growth}

On the basis of CT image superpixel gridding, based on the superpixel processing to improve the region growth criterion, the target edge of CT image is obtained and used as the initial contour to lay the foundation for accurate image segmentation $[22,23]$.

This section introduces the production criteria to calculate the CT image saliency map to obtain the image target edge. Although the region growth criterion only considers the pixel brightness, there is a problem that the shadow image segmentation effect is not good [24]. This section is based on superpixels to make up for the deficiency of the region growth criterion and incorporates the texture features of superpixels to improve the accuracy of region growth.

The energy feature, entropy $E$ feature, inertia $H$ feature, and correlation $K$ feature are, respectively, $C$ used as edge superpixel texture features, combined with gray level features to calculate the similarity of superpixels; the formula is as follows:

$$
T=\sqrt{\left(E_{i}-E_{j}\right)^{2}+\left(H_{i}-H_{j}\right)^{2}+\left(K_{i}-K_{j}\right)^{2}+\left(C_{i}-C_{j}\right)^{2}+\left(F_{i}-F_{j}\right)^{2}},
$$

where $T$ represents the similarity between $i$ the first pixel and $j$ the first pixel at the edge of the image, that is, the characteristic distance. $F$ indicates the average gray scale of the pixel.

According to the above formula, the feature distance between the organ pixel block and the background pixel block in the edge of the CT image is obtained, and the average feature distance is obtained by the statistical analysis principle, which can be used as the region growth criterion based on superpixels. Select a pixel block from the edge area of the image, take this pixel block as the center, determine whether the characteristic distance of its neighboring pixel blocks meets the growth criterion; if it meets, merge it, traverse all the pixel blocks according to the above method, and complete the growth. Get the growth map of the edge area of the CT image.

On the growth map of the edge area of the CT image, using the center-surround contrast idea [25] to calculate the edge salient area, the target edge can be obtained. The centersurround contrast idea is based on regional texture features and average gray levels. The texture feature distance of the edge region of the image is calculated according to the following formula, that is, the similarity of regional texture features:

$$
T^{\prime}=\sqrt{\left(E_{i}-E_{j}\right)^{2}+\left(H_{i}-H_{j}\right)^{2}+\left(K_{i}-K_{j}\right)^{2}+\left(C_{i}-C_{j}\right)^{2}} .
$$

Calculated the similarity weight of regional texture features according to the above $\beta$ formula:

$$
\beta=\frac{255}{T_{\max }},
$$

where ${ }^{\prime} T_{\max }$ is the maximum difference of the $T^{\prime}$ distance of the regional texture feature.

Calculate the texture feature distance between the current area and other areas. If the distance value of the current area is large, the area will be integrated into the current area. If the distance value is less than this area, the distance value of the current area will be used to subtract the distance value of the area $[26,27]$. Thus, get the global surround contrast of the current $M$ area:

$$
M=F^{\prime}+\chi \sum_{i=1} \beta T_{i}^{\prime}
$$

$F^{\prime}$ represents the gray value $\chi$ of the current area and the compensation coefficient. Under the condition of comparing the current area with different areas, it will change accordingly. The values are as follows:

$$
\chi= \begin{cases}1 & F^{\prime}>F_{\text {out }}, \\ -1 & F^{\prime} \leq F_{\text {out }} .\end{cases}
$$

$F_{\text {out }}$ represents the gray value of other areas.

Considering that there will be organs of a certain area in the actual CT image and may exist as edge locations, in order 
to clearly express the organ area, the global surround contrast weight should be set $\delta$ to calculate the image salience value. The calculation method is as follows:

$$
\delta=\frac{Z_{\text {back }}}{Z_{\text {org }}}
$$

$Z_{\text {back }}$ is the area of the background. $Z_{\text {org }}$ is the area of the organ area.

Combined with $\delta$ the weights, the final significance value can $M^{\prime}$ be calculated:

$$
M^{\prime}=\delta M
$$

According to the above formula, the salience values of all the edge regions are calculated, and the most salience region is selected; then, the target edge of the CT image can be obtained, which is used as the initial contour.

\section{The Proposed Algorithm}

Active contour model is the mainstream algorithm used in image segmentation in recent years. It combines level set and energy functional, while considering features such as image edges and regions, and has better performance than single feature segmentation algorithms $[28,29]$. Active contour models include many types. The Mumford-Shah model (the best approximation of the original image is found by piecewise smoothing function) is selected in this paper to improve CT image segmentation. Proceed as follows:

Input: CT image initial contour and superpixel information

Output: CT image improves segmentation results

Initialize the image superpixel and contour problem and use the Mumford-Shah model for final image segmentation, as follows:

(1) On the basis of the classic Mumford-Shah model, the level set method is used to solve the Mumford-Shah model and optimize it. This process is similar to curve evolution, transforming the energy function into a level set function, and the function solution value is obtained after continuous iteration. First, simplify the function of the Mumford-Shah model to obtain the energy functional:

$W\left(A, r_{\text {org }}, r_{\text {back }}\right)=M^{\prime}+\lambda \int_{\text {inside }(A)}\left(Q-r_{\text {org }}\right)^{2}+\lambda \int_{\text {outside }(A)}\left(Q-r_{\text {back }}\right)^{2}$,

where $V_{C C R}=K^{\prime} / K_{\text {total }}$ is the contour line, is the $\lambda$ edge level coefficient, is the $r_{\text {org }}$ target area feature, and is the $r_{\text {back }}$ background area feature and $Q$ is the image domain.

(2) Introduce the level $J(x, y, t)$ set function, solve the model, and obtain a new energy functional:

$$
\begin{aligned}
W^{\prime}= & J(x, y, t)+M^{\prime}+\lambda \int_{\text {inside }(J)}\left(Q-r_{\text {org }}\right)^{2} J(x, y, t) d x d y \\
& +\lambda \int_{\operatorname{outside}(J)}\left(Q-r_{\text {back }}\right)^{2} J(x, y, t) d x d y .
\end{aligned}
$$

(3) In order to make the segmentation result smoother, a Gaussian function convolution method is added to the energy function to convert the energy function into a signed distance function

(4) CT image segmentation is essentially the process of solving the target contour. In the Mumford-Shah model, it can also be regarded as the process of solving the minimum value of the energy functional (signed distance function), and the sign distance function of each pixel in the CT image is iterated. Use the initial contour obtained in the previous section to obtain the initial value of the $U_{0}$ symbolic distance function. Starting from the initialization of the symbolic distance function, the minimum value of the function is solved by traversal operation:

$$
U=U_{0}+\arg \min \left(W^{\prime}\right)
$$

(5) In this way, the superpixel grid segmentation of the CT image can be completed, and the final contour can be obtained

(6) End

Based on the above analysis, the flow of improved segmentation algorithm for superpixel grid CT image is given as shown in Figure 1.

\section{Experimental Results}

6.1. Experimental Environment and Data Set. In order to verify the effectiveness of the algorithm proposed in this paper, a comparative experiment was designed to highlight the performance of this algorithm. This experiment is run in the same environment, with $3.2 \mathrm{GHz} \mathrm{CPU}$, Intel i7-6700 as the central processing unit, Windows 10 as the computer operating system, and running in the experimental platform MATLAB.

The experimental data comes from the TCIA data set and DeepLesion data set.

TCIA data set. This data set is a large-scale medical. The medical data set contains various image data such as CT and MRI, with abundant clinical medical information

DeepLesion data set. It includes more than 32,000 lesion labels from more than 10,000 case studies, including image data of lesions in the lung, kidney, bone, lymph, and 


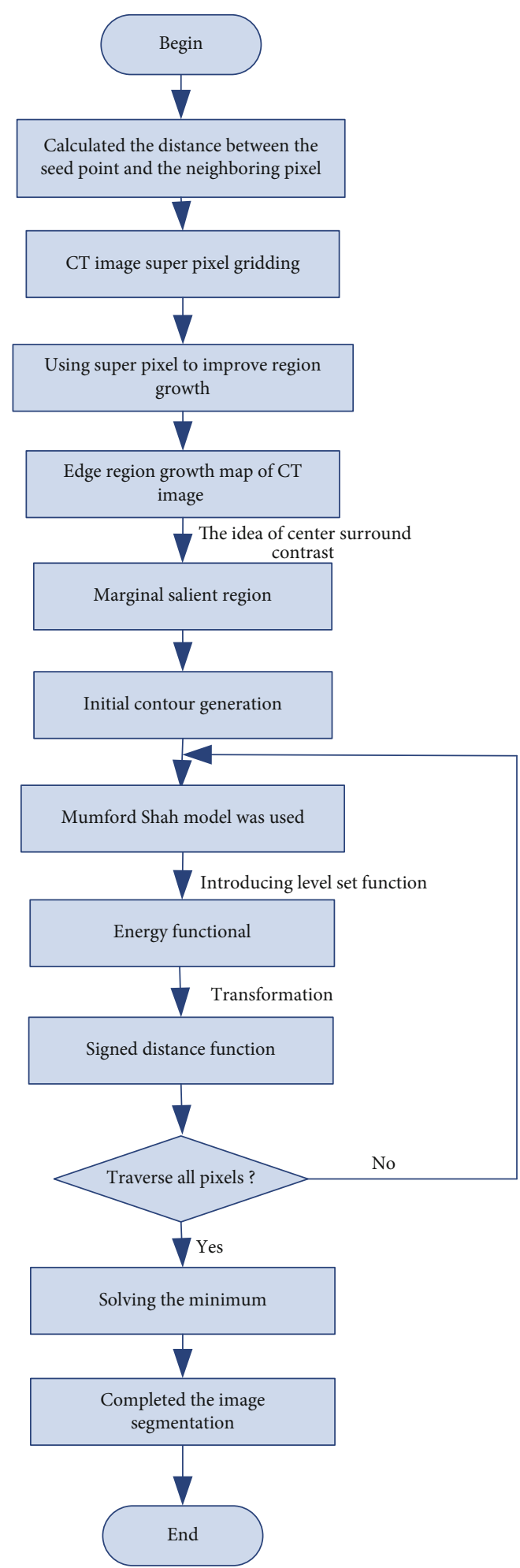

FIGURE 1: Flow of improved segmentation algorithm for superpixel grid CT image.

abdomen, a relatively comprehensive medical image data set currently released

The lung CT images, brain CT images, and vertebra CT images are selected from the above two data sets; 30 million are selected for each data set, for a total of 60 million data. Half of them are used as training data sets, and half are used as test data sets.

The $2 \times 2$ grid was used to input images, and lung CT images, brain CT images, and vertebral CT images were selected from TCIA data set and deep vision data set. Each data set selected 30 million, a total of 60 million data, of which 40 million were used as training data set, 10 million as verification data set, 10 million as validation data set, and 1000 as test data set.

\subsection{Experimental Steps}

(1) First installed basic operating software, including CPU acceleration software, combined with the experimental environment parameters set above, and run in the experimental platform MATLAB

(2) Select the superpixel gridding parameter by multiple experiments, that is, the number of pixel blocks $L$ to be divided. In this experiment, the final value of $L$ is selected within [1000-3000]

(3) Construct an improved Mumford-Shah model for training, input the test data set after the training is completed, and run the algorithm in the set experimental environment

(4) Repeat experiments to verify the effectiveness of the proposed algorithm

\subsection{Experimental Indicators}

(1) CT Image Segmentation Results and Manual Segmentation Results.

Taking the lung CT images, brain CT images, and vertebra CT images as the objects, respectively, the segmentation maps are drawn by computer, and at the same time, to verify the segmentation effect of the algorithm in this paper, compare the results with manual segmentation.

\section{(2) Superpixel Grid Time-Consuming: Superpixel Grid.}

Transformation is the prerequisite of image segmentation in this paper. This process requires constant iteration to find the clustering center, so time-consuming can be used as an important indicator to measure the performance of the algorithm.

(3) Image Target Edge Calculation Effect: According to the Public.

Equation (12) shows that $\delta$ the weight has an important influence on the accuracy of solving the saliency value. $\delta$ If the weight is selected properly, the image edge saliency can be calculated accurately. Therefore, the adaptability of $\delta$ the weight is selected to judge the accuracy of the algorithm image target edge calculation in this paper.

(4) Correct Classification Coefficient: Select the Correct Classification System. 


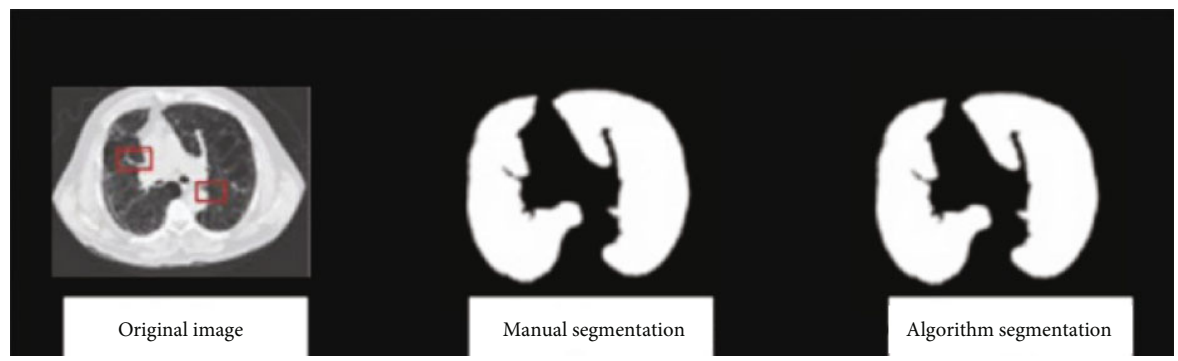

(a) CT image of the lung

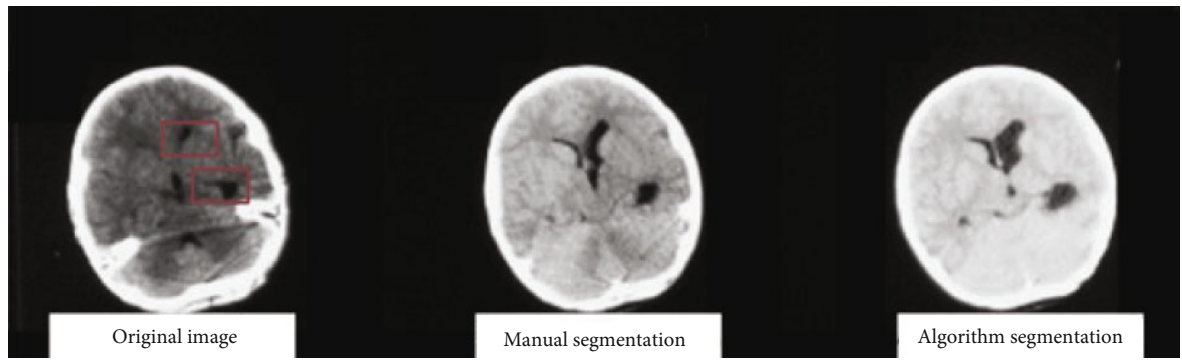

(b) CT image of the brain

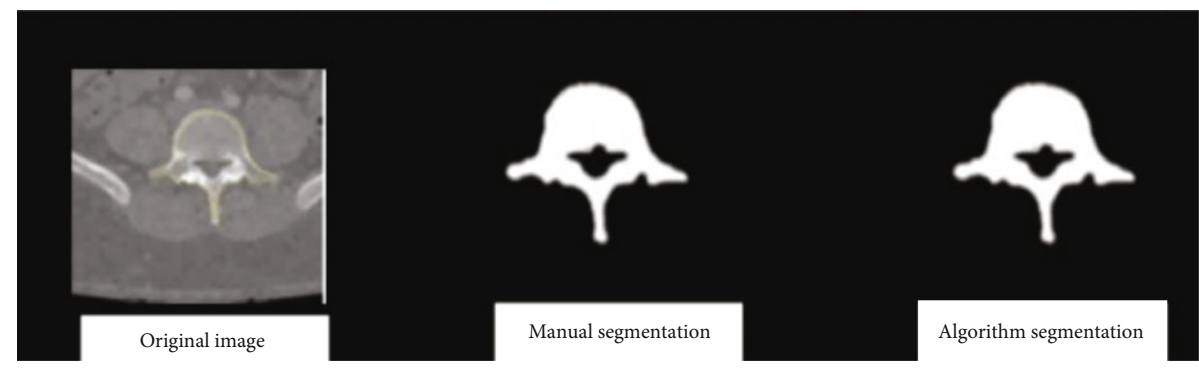

(c) CT images of the vertebrae

FIGURE 2: CT image segmentation results.

The $V_{\mathrm{CCR}}$ number is used as an indicator to judge the effect of algorithm segmentation, and the calculation formula is as follows:

$$
V_{\mathrm{CCR}}=\frac{K^{\prime}}{K_{\text {total }}},
$$

where $K^{\prime}$ represents the number of superpixels that are correctly $K_{\text {total }}$ segmented and represents the total number of image superpixels.

(5) Image Segmentation Accuracy: Segmented by CT Image.

The accuracy is the index to verify the performance of the algorithm, and the formula is as follows:

$$
\mathrm{SE}_{\mathrm{accu}}=\frac{L_{1}}{L_{\mathrm{tot}}} \times 100 \% \text {, }
$$

where $L_{\text {tot }}$ represents the number of pixel blocks $L_{1}$ actually divided and it represents the number of pixel blocks obtained by algorithmic division.
6.4. Results and Discussion. In the environment set in this experiment, the computer is used to draw the lung CT image, brain CT image, and vertebra CT image segmentation of the algorithm in this paper, as shown in the following Figure 2.

It can be seen from Figure 2 that the segmentation of lung CT images, brain CT images, and vertebra CT images using the algorithm in this paper is basically consistent with the manual segmentation results, and the segmentation effect is significant, and the accuracy is high, indicating that the algorithm in this paper has a good performance.

6.4.1. Experiment 2. Taking the time consumption of superpixel gridding as an indicator, compare the algorithm in this paper with the algorithm in literature [15-19]. To ensure the efficiency of the algorithm in this paper, the comparison results are shown in Table 1.

Analyzing the time-consuming of superpixel gridding of different algorithms in Table 1, it can be seen that as the number of pixel blocks increases, the time-consuming gridding increases, but the algorithm in this paper has a faster overall speed, with the highest time-consuming $0.8 \mathrm{~s}$, literature [19]. The algorithm has the highest time-consuming $2.5 \mathrm{~s}$ when the number of pixel blocks is 4000, the highest time-consuming of the algorithm in literature [16] and literature [17] reaches $2.0 \mathrm{~s}$, and the algorithm in literature [15] 
TABLE 1: Time-consuming comparison of superpixel gridding(s).

\begin{tabular}{lcccc}
\hline \multirow{2}{*}{ Algorithm } & \multicolumn{4}{c}{ Pixel block/piece } \\
& 1000 & 2000 & 3000 & 4000 \\
\hline Literature [15] algorithm & 0.5 & 0.8 & 1.2 & 1.6 \\
Literature [16] algorithm & 0.9 & 1.2 & 1.5 & 2.0 \\
Literature [17] algorithm & 1.2 & 1.6 & 1.8 & 2.0 \\
Literature [18] algorithm & 1.3 & 1.5 & 1.5 & 1.9 \\
Literature [19] algorithm & 0.8 & 1.5 & 1.9 & 2.5 \\
The proposed algorithm & 0.3 & 0.5 & 0.6 & 0.8 \\
\hline
\end{tabular}

TABLe 2: Fitness of weight $\delta$.

\begin{tabular}{lc}
\hline Experiment times/time & Fitness factor \\
\hline 50 & 0.66 \\
100 & 0.69 \\
150 & 0.75 \\
200 & 0.78 \\
250 & 0.80 \\
\hline
\end{tabular}

takes a short time when there are fewer pixel blocks. But with the increase in the number of pixel blocks, the timeconsuming increases rapidly, which is much higher than the algorithm in this paper. According to the data comparison, the CT image gridding effect of the algorithm in this paper is better, which is inseparable from the use of superpixels for analysis.

6.4.2. Experiment 3. Use the $\delta$ fitness of the weights for analysis. Usually, the fitness factor is higher than 0.5, which means the fitness is good. The fitness of the weights $\delta$ used in the algorithm in this paper is shown in Table 2.

Analysis of Table 2 shows that the weights used in the algorithm of this paper have a fitness higher $\delta$ than 0.5 in the course of many experiments, which proves that this paper has a good calculation effect on the salience value and can accurately complete the image edge acquisition, that is, the initial. The determination of the contour has certain accuracy, which lays the foundation for the determination of the final segmentation contour of the subsequent image. At the same time, it is proved that this paper uses the centersurround contrast idea to calculate the significant area of the image edge with a certain degree of reliability.

6.4.3. Experiment 4. The value range of $V_{\mathrm{CCR}}$ the correct classification coefficient is usually between $[0,1]$.

The higher the value, the better the algorithm performance. In order to verify the superior segmentation performance of the proposed algorithm, the proposed algorithm is compared with the literature [15-19]. It is shown in Figure 3.

According to the comparison chart of correct classification coefficient in Figure 2, it can be clearly found that the overall curve of the algorithm in this paper is higher than that of other literature algorithms, and the correct classification coefficient is as high as 0.9 , followed by the literature [16]

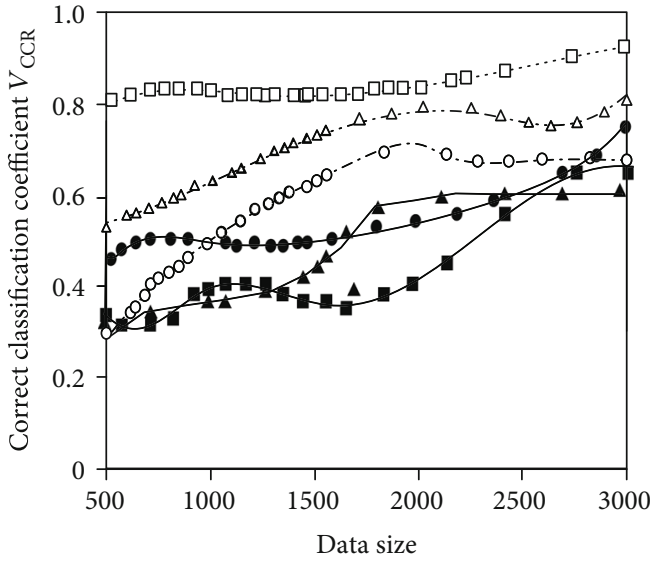

$\cdots \square$.. The proposed algorithm

- - - - Literature [15] algorithm

$\cdots \Delta \cdots$ Literature [16] algorithm

$\rightarrow-$ Literature [17] algorithm

- - Literature [18] algorithm

- - Literature [19] algorithm

FIGURE 3: Comparison of correct classification coefficients.

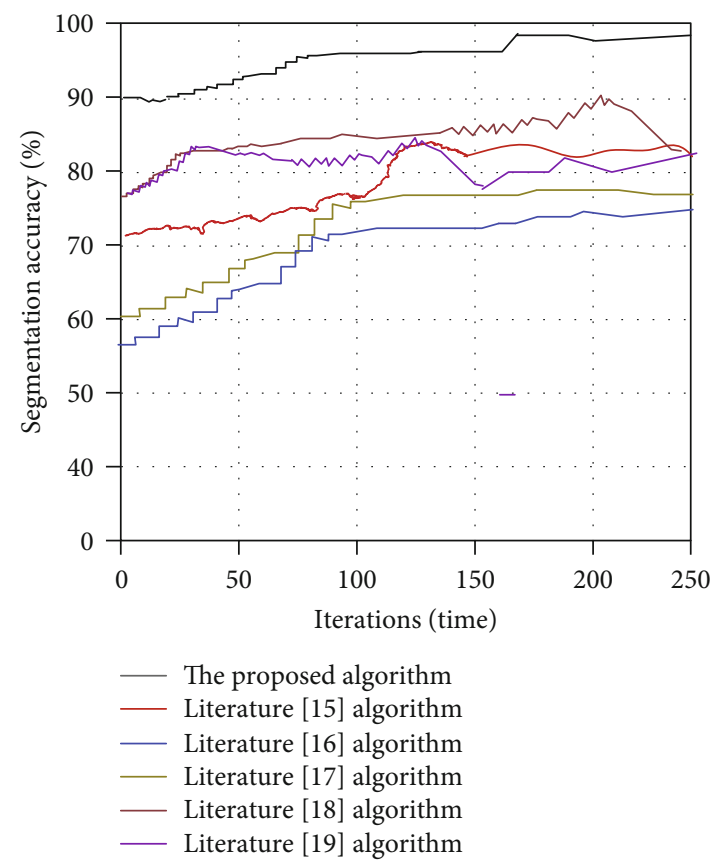

Figure 4: Comparison of segmentation accuracy.

algorithm. The correct classification coefficient is $[0.5,0.8]$. The algorithm of literature [17] has a maximum value of 0.7 , literature [15] has a rapid growth trend, but the maximum does not exceed 0.7 , and the maximum value of the remaining literature algorithms is below 0.7. The reason is that the algorithm in this paper first uses superpixels to improve the region growth criteria, calculates the salience value of the edge region of the image, and obtains the initial contour, which provides a basis for obtaining the final segmentation contour and improves the accuracy of pixel classification. 
6.4.4. Experiment Five. The comparison results of algorithm segmentation accuracy for CT images are shown in Figure 4.

According to the segmentation accuracy curve in Figure 4, it can be found that the algorithm in this paper has very obvious advantages, and the segmentation accuracy is always higher than 90\%. Among other documents, the average accuracy of literature [18] and literature [19] is above $80 \%$, followed by literature [15]. In comparison, literature [16] and literature [17] have lower image segmentation accuracy. The average level is around $70 \%$. Based on the initial contours obtained, this paper uses the level set method to improve the Mumford-Shah model in the active contour model. The improved active contour model is used for image segmentation, and the segmentation accuracy is higher.

\section{Conclusions}

The use of CT image detection to assist in clinical medical diagnosis has become the current mainstream. Image segmentation is an important step in image processing, and the results of segmentation play a cornerstone role in image three-dimensional reconstruction and other processing. At present, CT images are still a hot issue in medical image research. In order to obtain more accurate image segmentation results, this paper improves the active contour model and completes the superpixel grid CT image segmentation using the improved model. In the process of algorithm research, the problem of initial contour of the image is mainly considered, superpixels are used to improve the region growth criterion, the significant edge area is calculated, the initial contour is obtained, and the problem is solved by calculating the function based on the initial contour, and the final CT image segmentation is successfully completed. The results show that the proposed algorithm has high segmentation accuracy and short time-consuming, which provides a basis for CT image research.

However, this research still has certain shortcomings. The edge salient area obtained by using the region growth criterion and center-surround contrast can only get the largest salient area. In the future, a global saliency calculation method needs to be found to provide more efficient CT image segmentation. In addition, the processing of different types of medical images is also a direction that needs to be studied in the future to provide more useful help for medical diagnosis.

\section{Data Availability}

The data used to support the findings of this study are included within TCIA data set and DeepLesion data set.

\section{Conflicts of Interest}

The authors declare that they have no conflicts of interest.

\section{Acknowledgments}

This work is supported by the Heilongjiang Provincial Department of Education Natural Science Research Project
(No. 2016-KYYWF-0560) and the surface scientific and research projects of Jiamusi University (No. L2012-075).

\section{References}

[1] G. Ting, W. Weixing, L. Wei, and Y. Dandan, "Rock particle image segmentation based on improved normalized cut," International Journal of Control and Automation, vol. 10, no. 4, pp. 271-286, 2017.

[2] J. Xi, "Automatic spiking pulse-coupled neural network for image segmentation," International Journal of Future Generation Communication and Networking, vol. 10, no. 2, pp. 21-32, 2017.

[3] K. Srinivasa Rao, K. V. Satyanarayana, and P. Srinivasa Rao, "Segmentation of images using two parameter logistic type distribution and K-means clustering," International Journal of Grid and Distributed Computing, vol. 11, no. 12, pp. 1-20, 2018.

[4] G. L. F. da Silva, P. S. Diniz, J. L. Ferreira et al., "Superpixelbased deep convolutional neural networks and active contour model for automatic prostate segmentation on 3D MRI scans," Medical \& Biological Engineering \& Computing, vol. 58, no. 9, pp. 1947-1964, 2020.

[5] P. M. Gordaliza, A. Muñoz-Barrutia, M. Abella, M. Desco, S. Sharpe, and J. J. Vaquero, "Unsupervised CT lung image segmentation of a mycobacterium tuberculosis infection model," Scientific Reports, vol. 8, no. 1, pp. 9802-9808, 2018.

[6] X. Li, J. Sui, and Y. Wang, "Three-dimensional reconstruction of fuzzy medical images using quantum algorithm," IEEE Access, vol. 8, pp. 218279-218288, 2020.

[7] R. B. Holmes, I. S. Negus, S. J. Wiltshire, G. C. Thorne, P. Young, and The Alzheimer's Disease Neuroimaging Initiative, "Creation of an anthropomorphic CT head phantom for verification of image segmentation," Medical Physics, vol. 47, no. 6, pp. 2380-2391, 2020.

[8] M. Hamghalam and A. Ayatollahi, "White blood cell segmentation in Giemsa-stained images of blood smears," International Journal of Advanced Science and Technology, vol. 140, pp. 1-10, 2020.

[9] H. Transformation, V.-J. Algorithm, Indriyani, and I. M. Sudarma, "Automatic segmentation of U-zone area on facial images using fuzzy edge detection," International Journal of Advanced Science and Technology, vol. 133, pp. 19-30, 2019.

[10] A. A. Taha and A. Hanbury, "Metrics for evaluating 3D medical image segmentation: analysis, selection, and tool," $B M C$ Medical Imaging, vol. 15, no. 29, pp. 1-28, 2015.

[11] G. Chengcheng, Y. Fengqin, and C. Ying, "Image semantic segmentation based on convolutional neural network features and improved superpixel matching," Progress in Laser and Optoelectronics, vol. 55, no. 8, pp. 229-235, 2018.

[12] L. Jiarui, L. Cong, L. Xianjin, L. Wei, L. Ke, and H. Xinchao, "Image copy-paste tampering detection based on superpixel segmentation," Journal of Applied Sciences, vol. 37, no. 3, pp. 419-426, 2019.

[13] L. Miao, L. Yang, Z. Yuqian, and L. Yizhi, "A new method of image superpixel segmentation," Journal of Electronics and Information Technology, vol. 42, no. 2, pp. 83-89, 2020.

[14] T. Yongpeng, J. Yu, and X. Cong, "CT image segmentation method combining superpixels and CNN," Computer Engineering and Applications, vol. 56, no. 5, pp. 1-8, 2019. 
[15] L. Xia, G. Quan, L. Xiao, and W. Bo, "Joint energy active contour CT image segmentation method based on superpixels," Optoelectronic Engineering, vol. 47, no. 1, pp. 10-19, 2020.

[16] Q. Yan, W. Benzheng, Y. Yilong, C. Peipei, and C. Jinyu, "Lung parenchymal CT image refinement segmentation," Journal of Image and Graphics, vol. 22, no. 1, pp. 137-145, 2017.

[17] P. P. Rebouças Filho, P. C. Cortez, A. C. da Silva Barros, V. H. Albuquerque, and J. M. Tavares, "Novel and powerful $3 \mathrm{D}$ adaptive crisp active contour method applied in the segmentation of CT lung images," Medical Image Analysis, vol. 35, no. 2, pp. 503-516, 2017.

[18] L. Cong, S. Ding, L. Wang, A. Zhang, and W. Jia, "Image segmentation algorithm based on superpixel clustering," IET Image Processing, vol. 12, no. 11, pp. 2030-2035, 2018.

[19] N. Kishorjit Singh, N. Johny Singh, and W. Kanan Kumar, "Image classification using SLIC superpixel and FAAGKFCM image segmentation," IET Image Processing, vol. 14, no. 3, pp. 487-494, 2020.

[20] A. Ray, I. K. Maitra, and D. Bhattacharyya, "Detection of cervical cancer at an early stage using hybrid segmentation techniques from PAP smear images," International Journal of Advanced Science and Technology, vol. 112, pp. 23-32, 2018.

[21] M. V. Eijnatten, R. V. Dijk, J. Dobbe, G. Streekstra, J. Koivisto, and J. Wolff, "CT image segmentation methods for bone used in medical additive manufacturing," Medical Engineering \& Physics, vol. 10, no. 2, pp. 24-25, 2017.

[22] Y. Yin, Q. Huang, H. Gao, and Y. Xu, "Personalized APIs recommendation with cognitive knowledge mining for industrial systems," IEEE Transactions on Industrial Informatics, p. 1, 2020.

[23] Y. Yin, Z. Cao, Y. Xu, H. Gao, R. Li, and Z. Mai, "QoS prediction for service recommendation with features learning in mobile edge computing environment," IEEE Transactions on Cognitive Communications and Networking, vol. 6, no. 4, pp. 1136-1145, 2020.

[24] Y. Li, G. Cao, Q. Yu, and X. Li, "Active contours driven by non-local Gaussian distribution fitting energy for image segmentation," Applied Intelligence, vol. 48, no. 12, pp. 48554870, 2018.

[25] K. Zhang, L. Zhang, K.-M. Lam, and D. Zhang, "A level set approach to image segmentation with intensity inhomogeneity," IEEE Transactions on Cybernetics, vol. 46, no. 2, pp. 546-557, 2016.

[26] Z. Zhou, M. M. R. Siddiquee, N. Tajbakhsh, and J. Liang, "UNet++: redesigning skip connections to exploit multiscale features in image segmentation," IEEE Transactions on Medical Imaging, vol. 39, no. 6, pp. 1856-1867, 2020.

[27] X. Liu, S. Guo, B. Yang et al., "Automatic organ segmentation for CT scans based on super-pixel and convolutional neural networks," Journal of Digital Imaging, vol. 31, no. 5, pp. 748$760,2018$.

[28] M. F. Bobo, S. Bao, Y. Huo et al., "Fully convolutional neural networks improve abdominal organ segmentation," in Proceedings of SPIE-The International Society for Optical Engineering, pp. 100-121, United States, March 2018.

[29] W. Y. Sun, E. Q. Dong, Z. L. Cao, and Q. Zheng, "A robust local segmentation method based on fuzzy-energy based active contour," Acta Automatica Sinica, vol. 43, no. 4, pp. 611-621, 2017. 